\title{
Does Short-Term Dialysis Significantly Increase Coronary Artery Disease Burden in Diabetic Patients Who Undergo Renal Transplantation?
}

\author{
Umut Karabulut', Kudret Keskin ${ }^{2}$ \\ 'Department of Cardiology, Acıbadem International Hospital, ${ }^{2}$ Department of Cardiology, İstanbul Şişli Hamidiye Training and Research Hospital, i̇stanbul, Turkey \\ ORCID: \\ Umut Karabulut: 0000-0002-3947-9173 \\ Kudret Keskin: 0000-0002-9049-1530
}

\section{Abstract}

Background: Although preemptive renal transplantation decreases mortality associated with dialysis, coronary artery disease (CAD) remains the primary cause of mortality even after transplantation in patients with diabetes. We sought to determine whether short-term dialysis treatment significantly impacts CAD burden, revascularization strategy, and all-cause long-term mortality in diabetic renal transplant (RT) recipients without prior CAD. Subjects and Methods: Diabetic patients with end-stage renal disease and without prior CAD who were referred to coronary angiography before renal transplantation were retrospectively included. These patients were then divided into two groups as short-term dialyzed (nonpreemptive) and preemptive group. Angiographic findings, the severity of CAD, and long-term mortality were compared between the groups. Results: Overall, 164 included patients were included, of whom 125 (78\%) were male, and the median age was 54 years $(\mathrm{Q} 1-\mathrm{Q} 3=45-59)$. The mean duration of dialysis before RT was 1 year (range, $0.5-1.5$ years) in the nonpreemptive group. The extent of CAD, revascularization rates, SYNTAX, and Gensini scores were similar between groups (all $P>0.05$ ). During 4.8 years of follow-up, there were no significant differences in major adverse cardiovascular and cerebrovascular events ([hazard ratio $(\mathrm{HR})=0.88(0.38-2.01)$, $P=0.76])$ and all-cause mortality rates $([(\mathrm{HR})=0.59(0.20-1.71), P=0.33])$. Only age and hyperlipidemia were predictive of all-cause mortality $(\mathrm{HR}=1.03$ [1.001-1.07], $P=0.04$ and $\mathrm{HR}=2.75$ [1.20-6.28], $P=0.01$, respectively). Conclusion: Short-term dialysis does not seem to increase newly diagnosed CAD prevalence and burden in diabetic patients undergoing renal transplantation compared to patients who directly undergo renal transplantation. Moreover, long-term all-cause mortality rates did not differ between the two groups as well. Age and hyperlipidemia were independent predictors of all-cause mortality

Keywords: Coronary artery disease, diabetes, dialysis, renal transplantation

\section{INTRODUCTION}

Patients with end-stage renal disease (ESRD) have an increased risk of severe cardiac events such as myocardial infarction (MI) and death. In these patients, extensive coronary atherosclerosis may develop because of a high prevalence of risk factors such as diabetes and hypertension and the existence of pro-atherogenic factors associated with dialysis and renal failure. ${ }^{[1]}$ Moreover, as these patients age and their time spent on dialysis increases, so do the numbers of pre- and posttransplant cardiovascular events. ${ }^{[2]}$ Survival and quality of

Received: 18-04-2021 Revised: 30-05-2021 Accepted: 15-06-2021

Published Online: 25-09-2021

\section{Access this article online}

\section{Quick Response Code:}

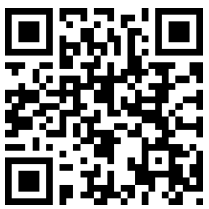

Website:

http://www.ijcva.com

DOI:

10.4103/ijca.ijca_17_21 life are superior in patients with ESRD who receive a renal transplant (RT) than those on dialysis. ${ }^{[3]}$ However, although survival in patients with ESRD improves considerably after $\mathrm{RT}$, the 10-year life expectancy is still worse than that in the general population.

Coronary artery disease (CAD) is the primary cause of morbidity and mortality after RT and is responsible for approximately $30 \%-50 \%$ of all deaths. In a recent report, cardiovascular

Address for correspondence: Dr. Umut Karabulut, Department of Cardiology, Acıbadem International Hospital, i̇stanbul, Turkey. E-mail: umkarabulut@gmail.com

This is an open access journal, and articles are distributed under the terms of the Creative Commons Attribution-NonCommercial-ShareAlike 4.0 License, which allows others to remix, tweak, and build upon the work non-commercially, as long as appropriate credit is given and the new creations are licensed under the identical terms.

For reprints contact: WKHLRPMedknow_reprints@wolterskluwer.com

How to cite this article: Karabulut U, Keskin K. Does short-term dialysis significantly increase coronary artery disease burden in diabetic patients who undergo renal transplantation? Int J Cardiovasc Acad 2021;7:63-9. 
disease, including angina pectoris, cerebrovascular accidents, and peripheral vascular disease, was present in $25 \%$ of patients at 10 years and $53 \%$ of those at 15 years after RT. In addition to traditional cardiovascular risk factors, factors related to ESRD, graft function, and immunosuppressive medication post-RT are also risk factors for CAD. ${ }^{[4,5]}$

Kidney disease is one of the most frequent complications of diabetes and is defined as a chronic renal disease with no other documented cause. ${ }^{[6]}$ Diabetes is still the leading cause of ESRD in most countries. ${ }^{[7]}$ Preemptive RT decreases morbidity and mortality associated with dialysis and reduces costs. Moreover, allograft failure is lower in patients who receive a preemptive transplant from a living donor than in those who do not. ${ }^{[8]}$ Patients with diabetes and ESRD who undergo preemptive transplantation have a two-fold higher risk of mortality than their nondiabetic counterparts. Patients with diabetes who had been waiting for transplantation for more than 2 years were found to be at a four-fold higher risk for mortality. ${ }^{[9]}$ Therefore, diabetes status is critical in RT recipients. Recent studies revealed that mortality is higher in patients waiting on dialysis than in preemptive RT recipients. Moreover, the duration of dialysis is known to be associated with survival after renal transplantation. Several studies have shown that long-term dialysis before transplantation is a strong predictor of posttransplant mortality. ${ }^{[9-11]}$ However, mortality data in comparative studies of patients undergoing short-term ( $\leq 1$ year) dialysis and preemptive transplantation are conflicting. ${ }^{[12,13]}$ Studies of the relationship between CAD, the pretransplant approach, and long-term survival are limited in RT candidates with diabetes. Since long-term hemodialysis is associated with adverse cardiac events and all-cause mortality, preemptive renal transplantation has become the preferred treatment of choice, if available. Nevertheless, it is not always feasible in real-world practice to perform preemptive transplantation in a timely fashion. Therefore, we sought to determine whether short-term dialysis significantly impacts CAD burden, revascularization strategy, and all-cause long-term mortality in diabetic RT recipients without prior CAD. In this way, we examined the effect of the treatment strategy used for CAD and the approach before transplantation on survival in transplant recipients with diabetes.

\section{Subjects and Methods}

This retrospective single-center study included 164 patients with diabetes and ESRD who were 18 years or older and underwent coronary angiography before RT between 2012 and 2020. Ethical approval for the study was obtained from the Bakırköy Dr. Sadi Konuk Training and Research Hospital Regional Ethics Committee (No: 2020-16). All participants' rights were protected, and written informed consent was obtained before the procedures, according to the Helsinki Declaration (2013).

Patients with a history of CAD, percutaneous coronary intervention, or coronary bypass surgery before angiography and those with advanced left ventricular systolic dysfunction (ejection fraction $<40 \%$ ) were excluded. Preemptive RT was defined as transplantation performed before the initiation of dialysis. Coronary angiography was performed before RT in patients with a positive cardiac stress test (treadmill test or myocardial perfusion scintigraphy). Finally, coronary angiography was performed in 136 patients due to a positive stress test and in 28 patients due to poor glycemic control (HbA1c $>7 \%)$. All nonpreemptive patients have already being dialyzed before angiography, and any preemptive (nondialyzed) patients have never been dialyzed before angiography or started after angiography. There is no angiographic follow-up after kidney transplantation.

Demographic data, laboratory findings, cardiovascular risk factors, insulin dependence status, and duration of hemodialysis before RT were obtained from the transplantation department database.

The patients were divided into a nondialysis (preemptive RT) group and a dialysis (nonpreemptive RT) group. Only 5\% of the patient's choice was peritoneal dialysis. Therefore, the type of dialysis was not used in regression analysis models. Critical CAD was defined as stenosis $\geq 70 \%$ in a significant coronary artery or major side branch $(>1.5 \mathrm{~mm}$ in diameter and $>20 \mathrm{~mm}$ length in lesion). Based on the results of coronary angiography, the following three distinct definitions were made: normal coronary arteries, noncritical CAD $(<70 \%)$, and critical CAD ( $\geq 70 \%)$. Localization of stenosis and the number of stenotic vessels were also recorded.

The Gensini and Synergy between percutaneous coronary intervention with Taxus and cardiac surgery (SYNTAX) scores were calculated for all patients to determine the extent and severity of CAD. Both scores were evaluated by two expert operators blinded to the angiography results. When there was a discrepancy between the two experts, an opinion was sought from a third cardiologist. Procedural details, including stent type, size, and diameter, were also recorded for patients who underwent coronary revascularization. Short-term dialysis was defined as dialysis with a duration of 1-18 months (<1.5 years). Major adverse cardiovascular and cerebrovascular adverse events (MACCE) were defined as the composite outcome of nonfatal $\mathrm{MI}$, unstable angina pectoris (USAP), nonfatal stroke, urgent revascularization, cardiac hospitalization, and cardiac mortality. The endpoints of the study were major adverse cardiac and cerebrovascular events (MACCE) and all-cause mortality.

\section{Statistical analysis}

Continuous variables are shown as the median and interquartile range, and categorical variables as the number and percentage. The distribution of data was assessed using the Kolmogorov-Smirnov test. Continuous variables were compared between groups using an independent-sample $t$-test or Mann-Whitney U-test, as appropriate. Categorical data were compared using the Chi-square test or Fisher's exact test. Survival curves were generated using the Kaplan-Meier 
method. Differences in the survival curves were assessed using the log-rank test. Cox regression analysis was used to determine the factors related to survival. Variables found to be significant in univariate analysis or with clinical relevance were included in the multivariate analysis. All statistical analyses were conducted using SPSS software version 22 (IBM Corp., Armonk, NY, USA). A two-tailed $P<0.05$ was considered statistically significant.

\section{Ethical Statement}

Ethical approval for the study was obtained from the Bakırköy Dr. Sadi Konuk Training and Research Hospital Regional Ethics Committee (No: 2020-16). All participants' rights were protected, and written informed consent was obtained before the procedures, according to the Helsinki Declaration (2013).

\section{RESULTS}

A total of 164 patients were included in the study. One hundred and twenty-five (78\%) patients were male. The patient characteristics at baseline are shown in Table 1. There was no statistically significant between-group difference in the rates of insulin-dependent diabetes mellitus $(66 \%, n=109)$, hypertension $(58.5 \%, n=96)$, hyperlipidemia $(31.1 \%, n=51)$, or cigarette smoking $(15.9 \%, n=26)$. The mean creatinine, potassium, calcium, albumin, and hemoglobin levels were $6.6 \mathrm{mg} / \mathrm{dL}, 5.1 \mathrm{mg} / \mathrm{dL}, 8.9 \mathrm{mg} / \mathrm{dL}, 3.8 \mathrm{mg} / \mathrm{dL}$, and $11.2 \mathrm{mg} /$ $\mathrm{dL}$, respectively; all these values were significantly higher in the nonpreemptive RT group $(P<0.001)$. The mean duration of dialysis before RT was 1 year (range, $0.5-1.5$ years) in the nonpreemptive group.

The angiographic characteristics of the patients are shown in Table 2. Noncritical CAD occurred in 22 patients $(17.9 \%)$ in the nonpreemptive group and nine $(22.0 \%)$ in the preemptive group; the between-group difference was not statistically significant $(P=0.56)$.

Single-vessel disease was found in 25 patients $(20.3 \%)$ in the nonpreemptive group and eight patients $(19.5 \%)$ in the preemptive group with respective rates of $7.3 \%(n=9)$ and $7.3 \%(n=3)$ for two-vessel disease and $21.3 \%(n=26)$ and $20.0 \%(n=8)$ for multi-vessel disease; there were no significant between-group differences $(P=0.91, P=1$, and $P=0.86$, respectively). Moreover, the SYNTAX and Gensini scores were not significantly different between the two groups ( $P=0.85$ and $P=0.68$, respectively).

Percutaneous coronary intervention was performed in $15.4 \%$ of patients $(n=19)$ in the nonpreemptive group and in $14.6 \%(n=6)$ in the preemptive group; coronary artery bypass graft surgery was performed in $17.1 \%(n=21)$ and $17.5 \%(n=7)$, respectively; there were no significant between-group differences ( $P=0.9$ and $P=0.95$, respectively). Furthermore, there was no significant difference in stent type, size, or length between the groups $(P=0.49, P=0.59$, and $P=0.09$, respectively).

Cox regression analysis was performed to determine the factors related to long-term survival. Variables found to be significant in univariate analysis or with clinical relevance were included in the multivariate analysis. The results of the Cox regression analysis for all-cause mortality are shown in Table 3. Factors found to be significantly related to survival in the univariate analysis were patient age $(P=0.04)$, hypertension $(P=0.04)$, hyperlipidemia $(P=0.001)$, and smoking $(P=0.02)$. Only patient age $(P=0.04)$ and hyperlipidemia $(P=0.01)$ were significantly related to survival in the multivariate

\begin{tabular}{|c|c|c|c|c|}
\hline & Total $(n=164)$ & Nonpreemptive TX ( $n=123,75 \%)$ & Preemptive TX ( $n=41,25 \%)$ & $P$ \\
\hline Age (years), median (IQR) & $54(45-59)$ & $54(44-60)$ & $54(46-57)$ & 0.94 \\
\hline Gender (male), $n(\%)$ & $128(78.0)$ & $95(77.2)$ & $33(80.5)$ & 0.66 \\
\hline IDDM, $n(\%)$ & $109(66.5)$ & $81(65.9)$ & $28(68.3)$ & 0.77 \\
\hline Hypertension, $n(\%)$ & $96(58.5)$ & $69(56.1)$ & $27(65.9)$ & 0.27 \\
\hline Hyperlipidemia, $n(\%)$ & $51(31.1)$ & $38(30.9)$ & $13(31.7)$ & 0.92 \\
\hline Smoking, $n(\%)$ & $26(15.9)$ & $20(16.3)$ & $6(14.6)$ & 0.80 \\
\hline Dialysis duration (years), median (IQR) & - & $1.0(0.5-1.5)$ & - & N/A \\
\hline Normal coronary arteries, $n(\%)$ & $51(31.1)$ & $41(33.3)$ & $10(24.4)$ & 0.28 \\
\hline Multi-vessel disease, $n(\%)$ & $34(21.0)$ & $26(21.3)$ & $8(20.0)$ & 0.86 \\
\hline Glucose (mg/dl), median (IQR) & $139(113-184)$ & $144(119-192)$ & $133(101-175)$ & 0.09 \\
\hline HbA1c (\%), median (IQR) & $7.1(6.3-8.0)$ & $7.2(6.4-8.2)$ & $7.0(6.1-7.5)$ & 0.16 \\
\hline Creatinine (mg/dl), median (IQR) & $6.6(5.3-8.1)$ & $6.9(5.7-8.7)$ & $5.5(5.0-6.9)$ & $<0.001$ \\
\hline Sodium, median (IQR) & $137(135-139)$ & $137(135-139)$ & $138(135-140)$ & 0.10 \\
\hline Potassium, median (IQR) & $5.1(4.6-5.5)$ & $5.3(4.7-5.7)$ & $4.8(4.3-5.1)$ & $<0.001$ \\
\hline Calcium, median (IQR) & $8.9(8.5-9.4)$ & $9.0(8.6-9.5)$ & $8.6(8.3-8.9)$ & $<0.001$ \\
\hline Uric acid median (IQR) & $5.6(4.4-6.8)$ & $5.5(4.3-6.6)$ & $6.4(4.8-7.6)$ & 0.006 \\
\hline Albumin median (IQR) & $3.8(3.5-4.3)$ & $3.9(3.6-4.3)$ & $3.4(3.0-3.7)$ & $<0.001$ \\
\hline Hemoglobin (gr/dL), median (IQR) & $11.2(10.0-12.5)$ & $11.6(10.4-12.9)$ & $9.7(8.7-10.8)$ & $<0.001$ \\
\hline WBC $\left(\times 10^{3}\right)$, median (IQR) & $7.7(6.6-9.2)$ & $7.8(6.6-9.1)$ & $7.6(6.8-9.9)$ & 0.51 \\
\hline Platelet $\left(\times 10^{3}\right)$, median $(\mathrm{IQR})$ & $228(190-280)$ & $226(184-276)$ & $231(193-315)$ & 0.39 \\
\hline
\end{tabular}

IDDM: Insulin dependent diabetes mellitus, WBC: White blood count, IQR: Interquartile range, HbA1c:HemoglobinA1c, N/A: Not applicable, TX: Transplantation 
Table 2: Angiographic characteristics of the study patients

\begin{tabular}{|c|c|c|c|c|}
\hline & Total $(n=164)$ & Nonpreemptive TX ( $n=123,75 \%)$ & Preemptive TX $(n=41,25 \%)$ & $P$ \\
\hline Normal coronary arteries, $n(\%)$ & $51(31.1)$ & $41(33.3)$ & $10(24.4)$ & 0.28 \\
\hline Non-critical CAD, $n(\%)$ & $31(18.9)$ & $22(17.9)$ & $9(22.0)$ & 0.56 \\
\hline Single vessel disease, $n(\%)$ & $33(20.1)$ & $25(20.3)$ & $8(19.5)$ & 0.91 \\
\hline Two vessel disease, $n(\%)$ & $12(7.3)$ & $9(7.3)$ & $3(7.3)$ & 1.00 \\
\hline Three vessel disease, $n \%$ & $34(21.0)$ & $26(21.3)$ & $8(20.0)$ & 0.86 \\
\hline Critical LAD stenosis, $n(\%)$ & $51(31.1)$ & $39(31.7)$ & $12(29.3)$ & 0.77 \\
\hline Critical CX stenosis, $n(\%)$ & $35(21.3)$ & $26(21.1)$ & $9(22.0)$ & 0.91 \\
\hline Critical RCA stenosis, $n(\%)$ & 49 (29.9) & $37(30.1)$ & $12(29.3)$ & 0.92 \\
\hline Critical side branch stenosis $n(\%)$ & $26(15.9)$ & $20(16.3)$ & $6(14.6)$ & 0.80 \\
\hline SYNTAX score, median (IQR) & $2.0(0-9.7)$ & $2.0(0-9.0)$ & $2.0(0-12.0)$ & 0.85 \\
\hline Gensini score, median (IQR) & $6.0(0-23.7)$ & $6.0(0-22.0)$ & $6.0(0-29.5)$ & 0.68 \\
\hline Percutaneous coronary intervention, $n(\%)$ & $25(15.2)$ & $19(15.4)$ & $6(14.6)$ & 0.90 \\
\hline DES, $n(\%)$ & $19(11.7)$ & $13(10.7)$ & $6(14.6)$ & 0.49 \\
\hline Stent diameter (mm), mean (SD) & $2.83(0.16)$ & $2.84(0.15)$ & $2.80(0.19)$ & 0.59 \\
\hline Stent length (mm), median (IQR) & $18(17-29)$ & $18(16-22)$ & $23(18-44)$ & 0.09 \\
\hline CABG, $n(\%)$ & $28(17.2)$ & $21(17.1)$ & $7(17.5)$ & 0.95 \\
\hline
\end{tabular}

CAD: Coronary artery disease, LAD: Left anterior descending, Cx: Circumflex, RCA: Right coronary artery, DES: Drug-eluting stent, CABG: Coronary artery by-pass graft, SD: Standard deviation, IQR: Interquartile range, SYNTAX: Synergy taxus and cardiac surgery, TX: Transplantation

Table 3: Cox regression analysis for all-cause mortality

\begin{tabular}{|c|c|c|c|c|}
\hline & Uni & & Mult & \\
\hline & HR (95\%Cl) & $P$ & HR (95\%Cl) & $P$ \\
\hline Preemptive approach & $0.59(0.20-1.71)$ & 0.33 & $0.51(0.17-1.50)$ & 0.22 \\
\hline Age & $1.03(1.001-1.075)$ & 0.04 & $1.03(1.001-1.07)$ & 0.04 \\
\hline Gender & $1.27(0.48-3.33)$ & 0.62 & & \\
\hline Hypertension & $2.28(1.03-5.05)$ & 0.04 & $1.28(0.54-3.02)$ & 0.56 \\
\hline Hyperlipidemia & $3.61(1.72-7.56)$ & 0.001 & $2.75(1.20-6.28)$ & 0.01 \\
\hline Insulin dependent diabetes & $2.1(0.83-5.76)$ & 0.11 & & \\
\hline Smoking & $2.72(1.14-6.48)$ & 0.02 & $1.98(0.79-4.95)$ & 0.14 \\
\hline Multivessel coronary disease & $1.42(0.60-3.34)$ & 0.41 & & \\
\hline Any critical stenosis & $1.85(0.88-3.86)$ & 0.10 & & \\
\hline $\mathrm{HbA1c}$ & $1.14(0.92-1.41)$ & 0.21 & & \\
\hline Creatinine & $0.95(0.79-1.14)$ & 0.63 & & \\
\hline Calcium & $0.85(0.56-1.29)$ & 0.45 & & \\
\hline Albumin & $0.85(0.46-1.54)$ & 0.59 & & \\
\hline Uric acid & $0.92(0.74-1.15)$ & 0.92 & & \\
\hline Hemoglobin & $0.97(0.86-1.10)$ & 0.71 & & \\
\hline
\end{tabular}

HbA1c: Hemoglobin A1c, CI: Confidence interval, HR: Hazard ratio

analysis. A total of 37 MACCE occurred during the follow up $(30[24.4 \%]$ in the nonpreemptive group and $7[17.1 \%]$ in the preemptive group), and there was no significant difference between the groups $(P=0.33)$ USAP, Nonfatal MI, Urgent Revascularization, Cardiac hospitalization, nonfatal stroke and cardiac mortality and all-cause mortality rates were similar $(P=1.00, P=1.00, P=0.70, P=0.79, P=0.43$, $P=0.27$ and 0,55 respectively) [Table 4].

A preemptive RT approach was not found to predict all-cause mortality $(P=0.33)$. The median follow-up duration was 4.8 years (interquartile range, $2.7-7.1$ years). The estimated mean survival time was 7.86 years (lower bound, 7.247; upper bound, 8.312) in the preemptive and 7.72 years (lower bound, 7.196; upper bound, 8.2) in the nonpreemptive group.
Kaplan-Meier analyses revealed no significant between-group differences in long-term all-cause mortality (hazard ratio $[\mathrm{HR}]=0.59,95 \%$ confidence interval $[\mathrm{CI}][0.20-1.71]$; $P=0.33)$ and MACCE $(\mathrm{HR}=0.88,95 \%$ CI $[0.38-2.01]$, $P=0.76)$ [Figures 1 and 2]. Twenty-nine $(17.7 \%)$ of the 164 patients in the study died in the long-term, with no significant difference in the death rate between the two groups (six [14.6\%] in the preemptive group vs. 23 [18.7\%] in the nonpreemptive group; $P=0.55)$. Seven $(24.1 \%)$ of the 29 patients who died had multivessel CAD, and $27(20.3 \%)$ of the 133 surviving patients had multivessel disease. There was no difference in the severity of CAD between the RT recipients who died and those who survived $(P=0.64)$. The cause of death among these 29 patients was MI in 13 (44.8\%), sudden cardiac 


\begin{tabular}{|c|c|c|c|c|}
\hline & Total $(n=164), n(\%)$ & Nonpreemptive TX ( $n=123,75 \%), n(\%)$ & Preemptive TX ( $n=41,25 \%), n$ (\%) & $P$ \\
\hline MACCE & $37(22.6)$ & $30(24.4)$ & $7(17.1)$ & 0.33 \\
\hline USAP & $8(4.9)$ & $6(4.9)$ & $2(4.9)$ & 1.00 \\
\hline Nonfatal MI & $5(3.0)$ & $4(3.3)$ & $1(2.4)$ & $1.00^{*}$ \\
\hline Urgent revascularization & $10(6.1)$ & $8(6.5)$ & $2(4.9)$ & 0.70 \\
\hline Cardiac hospitalization & $22(13.4)$ & $17(13.8)$ & $5(12.2)$ & 0.79 \\
\hline Nonfatal stroke & $2(1.2)$ & $1(0.8)$ & $1(2.4)$ & 0.43 \\
\hline Cardiac mortality & $20(12.2)$ & $17(13.8)$ & $3(7.3)$ & 0.27 \\
\hline All-cause mortality & $29(17.7)$ & $23(18.7)$ & $6(14.6)$ & 0.55 \\
\hline
\end{tabular}

*Fisher's exact test. MACCE: Major adverse cardiovascular and cerebrovascular events, USAP: Unstable angina pectoris, MI: Myocardial infarction, TX: Transplantation

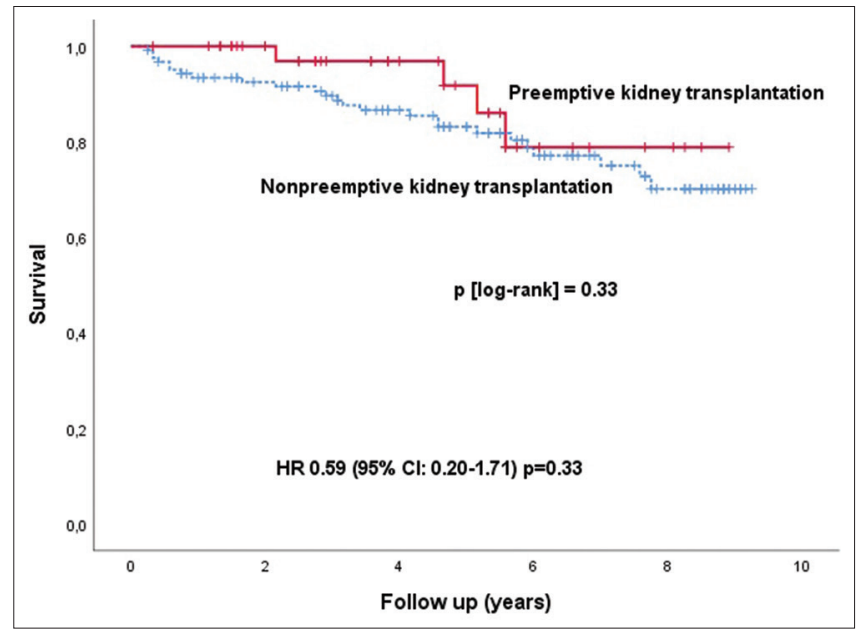

Figure 1: Kaplan-Meier analyses revealed no significant between-group difference in long-term all-cause mortality (hazard ratio 0.59, 95\% confidence interval $0.20-0.71 ; P=0.33$ )

death in four (13.7\%), sepsis in four (13.7\%), liver failure in two $(6.9 \%)$, cerebrovascular accident in two $(6.9 \%)$, kidney failure in one (3.4\%), pneumonia in one (3.4\%), lung cancer in one (3.4\%), and heart failure in one (3.4\%).

\section{Discussion}

In this study, we found that that the extent, severity, the need for revascularization for CAD, and long-term MACCE and mortality rates were similar between preemptive and short-term dialyzed RT recipients with diabetes. Thus, we believe that short-term dialysis did not significantly affect the incidence of newly diagnosed CAD compared to the preemptive approach.

Preemptive transplantation has been associated with improved graft survival and reduced mortality in recipients of both cadaveric and living donor transplants. ${ }^{[14]}$ An association between a longer dialysis duration before transplantation and higher cardiovascular disease rates and mortality has also been reported. ${ }^{[7]}$ There was no significant difference in long-term mortality between the preemptive and nonpreemptive RT groups in our study. We considered that this might be because of the short-term dialysis until transplantation in the nonpreemptive group. Most of the nonpreemptive recipients

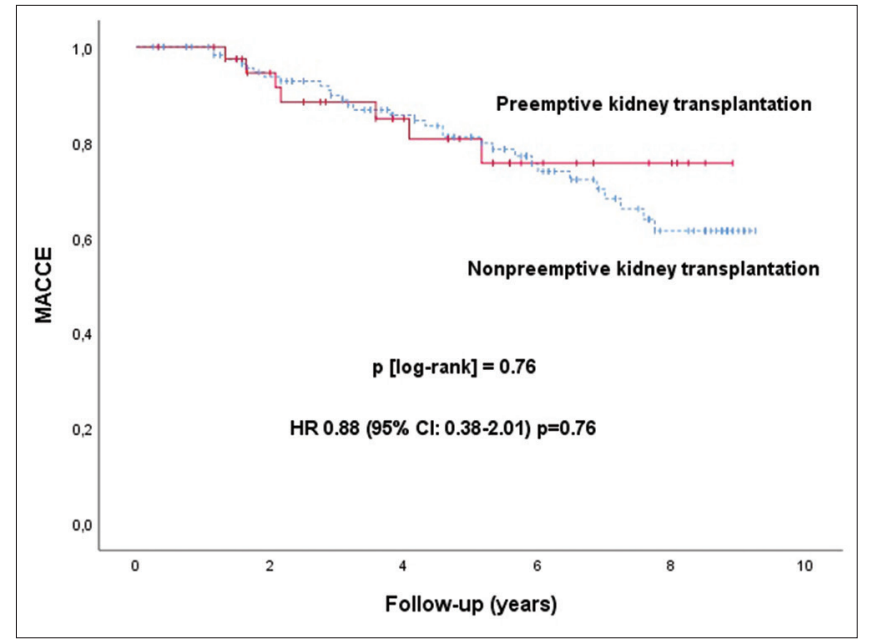

Figure 2: Kaplan-Meier analyses revealed no significant between-group difference in Major adverse cardiovascular and cerebrovascular adverse events (hazard ratio $0.88,95 \%$ confidence interval $0.38-2.01 ; P=0.76$ )

underwent RT within the $1^{\text {st }}$ year. Further, RTs in our country are mostly from living donors, which decreases the dialysis duration and waiting time to RT. A recent study found that the duration of dialysis predicted mortality in young and older RT patients. ${ }^{[15]}$ Haller et al. found that the potential beneficial effect of preemptive transplantation was reduced in more recent years. Moreover, long-term dialysis ( $>1$ year) was associated with higher mortality after RT. ${ }^{[16]}$ Another large cohort study showed that 6-11 months of dialysis before transplantation is not associated with a higher risk of mortality than $0-5$ months of dialysis. Our study findings are compatible with those of these two studies. ${ }^{[17]}$ As observed, the most important determinant of pretransplant dialysis's adverse effects not reflected in posttransplant mortality is the short-term duration. Moreover, the decrease in the need for transfusion provided by erythropoietin and iron therapy reduces HLA antibody sensitivity, and the widespread use of more effective and less toxic immunosuppressive treatments, and the gradual increase in the standards of care for dialysis patients are other important factors. In contrast to our study, a Japanese cohort study found that the long-term clinical event rate (death, cardiovascular disease, and graft loss) is significantly lower in preemptive RT recipients than in dialyzed patients irrespective of duration. ${ }^{[12]}$ 
However, the study's composite endpoint was different as it was an ethnicity-based cohort that may have resulted in different findings compared to a European population.

The extent and severity of CAD and the need for coronary revascularization and revascularization strategies did not differ between our study groups. These findings suggest that the effect of a relatively short dialysis duration on cardiac disease burden and long-term mortality is similar. We found that neither critical coronary stenosis nor multivessel disease was a predictor of long-term all-cause mortality. Different results were found in several studies examining the relationship between the severity of pretransplant CAD and long-term survival. Gowdak et al. found that mortality was significantly higher in patients with critical CAD by approximately 2 years of follow-up after kidney transplantation than patients with noncritical CAD. ${ }^{[18]}$ In contrast, other studies found that neither the severity of pretransplant CAD nor the treatment strategy significantly affects long-term mortality. ${ }^{[19,20]}$ Furthermore, Jones et al. examined long-term survival in patients with ESRD who underwent coronary angiography before transplantation. Unlike in our study, long-term survival in patients with single-vessel or multivessel disease was significantly lower than in noncritical patients. However, in multivariate analysis, the multivessel disease only was an independent predictor of long-term survival. ${ }^{[2]]}$ There may be several reasons why critical CAD did not predict all-cause mortality in our study. First, it may reflect the study's small sample size; second, all patients underwent angiography, and an unprecedented proportion underwent percutaneous coronary intervention or coronary artery bypass graft before RT. Another reason may be that those with a history of CAD (MI, percutaneous coronary intervention, and coronary artery bypass graft surgery) before angiography were excluded from the study. This may have affected the results because the total CAD burden decreased.

As expected, one of the independent predictors of long-term mortality was age, which is compatible with the findings of other studies..$^{[9,14,22]}$ Another independent predictor in our study was hyperlipidemia. Data regarding the effect of hyperlipidemia on mortality in RT recipients are conflicting. Jardine et al. found a significant relationship between a high cholesterol level and posttransplant cardiac death in univariate analysis but not multivariate analysis. ${ }^{[23]}$ In another large study, a significant decrease in the frequency of major adverse cardiovascular events (death, nonfatal MI, and need for coronary intervention) was found in the lower low-density lipoprotein cholesterol group. Still, the all-cause mortality rate was similar to that in the placebo group. ${ }^{[24]}$

\section{Limitations of the study}

First single-center and retrospective design and second relatively small sample size. However, these shortcomings were, to some extent, offset by our long follow-up duration. Furthermore, we could not able to find out and compare the lipid parameters of the patients, this may be another limitation.

\section{Conclusion}

This study found that short-term dialysis before RT and newly diagnosed CAD do not negatively impact long-term MACCE and survival in diabetic RT recipients without a previous CAD history compared with preemptive RT. Moreover, the prevalence and severity of CAD were similar between the groups.

\section{Declaration of patient consent}

The authors certify that they have obtained all appropriate patient consent forms. In the form the patients have given their consent for their images and other clinical information to be reported in the journal. The patients understand that their names and initials will not be published and due efforts will be made to conceal their identity, but anonymity cannot be guaranteed.

\section{Acknowledgments}

We would like to express our gratitude to the kidney transplant coordinator Derya Candan, the assistant coordinator Gamze Dedebey, transplant nurse Nilgün Demiröz and the chief of the Kidney Transplant and Nephrology department, Ülkem Çakır Professor MD., who supported us in the data extraction of this article.

\section{Financial support and sponsorship \\ Nil.}

\section{Conflicts of interest}

There are no conflicts of interest.

\section{RefERENCES}

1. Foley RN, Parfrey PS, Sarnak MJ. Clinical epidemiology of cardiovascular disease in chronic renal disease. Am J Kidney Dis 1998;32:S112-9.

2. Jeloka TK, Ross H, Smith R, Huang M, Fenton S, Cattran D, et al. Renal transplant outcome in high-cardiovascular risk recipients. Clin Transplant 2007;21:609-14.

3. Wolfe RA, Ashby VB, Milford EL, Ojo AO, Ettenger RE, Agodoa LY, et al. Comparison of mortality in all patients on dialysis awaiting transplantation, and recipients of a first cadaveric transplant. N Engl J Med 1999;341:1725-30.

4. Vanrenterghem YF, Claes K, Montagnino G, Fieuws S, Maes B, Villa M, et al. Risk factors for cardiovascular events after successful renal transplantation. Transplantation 2008;85:209-16.

5. Lentine KL, Brennan DC, Schnitzler MA. Incidence and predictors of myocardial infarction after kidney transplantation. J Am Soc Nephrol 2005;16:496-506.

6. Kidney Disease Improving Global Outcomes. KDIGO 2012 Clinical Practice Guideline for the evaluation and management of chronic kidney disease. Kidney Int Suppl 2013;3:1-150.

7. Atkins RC. The epidemiology of chronic kidney disease. Kidney Int Suppl 2005;94:S14-8.

8. Mange KC, Joffe MM, Feldman HI. Effect of the use or nonuse of long-term dialysis on the subsequent survival of renal transplants from living donors. N Engl J Med 2001;344:726-31.

9. Meier-Kriesche HU, Port FK, Ojo AO, Rudich SM, Hanson A, Cibrik DM, et al. Effect of waiting time on renal transplant outcome. Kidney Int 2000;58:1311-7.

10. Aufhauser DD Jr., Peng AW, Murken DR, Concors SJ, Abt PL, Sawinski D, et al. Impact of prolonged dialysis prior to renal transplantation. Clin Transplant 2018;32:e13260.

11. Prezelin-Reydit M, Combe C, Harambat J, Jacquelinet C, Merville P, Couzi L, et al. Prolonged dialysis duration is associated with graft 
failure and mortality after kidney transplantation: Results from the French transplant database. Nephrol Dial Transplant 2018;34:1-7.

12. Goto N, Okada M, Yamamoto T, Tsujita M, Hiramitsu T, Narumi S, et al. Association of dialysis duration with outcomes after transplantation in a Japanese cohort. Clin J Am Soc Nephrol 2016;11:497-504.

13. Unsal MG, Yilmaz M, Sezer T, Celtik A, Unalp OV, Uguz A, et al. Comparison of preemptive kidney transplantation with nonpreemptive kidney transplantation in a single center: A follow-up study. Transplant Proc 2015;47:1385-7.

14. Kasiske BL, Snyder JJ, Matas AJ, Ellison MD, Gill JS, Kausz AT. Preemptive kidney transplantation: The advantage and the disadvantaged. J Am Soc Nephrol 2002;13:1358-64.

15. Ma MK, Lim WH, Craig JC, Russ GR, Chapman JR, Wong G. Mortality among younger and older recipients of kidney transplants from expanded criteria donors compared with standard criteria donors. J Am Soc Nephrol 2016;11:128-36.

16. Haller MC, Kainz A, Baer H, Oberbauer R. Dialysis vintage and outcomes after kidney transplantation: A retrospective cohort study. Clin J Am Soc Nephrol 2017;12:122-30.

17. Helanterä I, Salmela K, Kyllönen L, Koskinen P, Grönhagen-Riska C, Finne P. Pretransplant dialysis duration and risk of death after kidney transplantation in the current era. Transplantation 2014;98:458-64.

18. Gowdak LH, de Paula FJ, César LA, Filho EE, Ianhez LE, Krieger EM, et al. Screening for significant coronary artery disease in high-risk renal transplant candidates. Coron Artery Dis 2007;18:553-8.

19. Hage FG, Smalheiser S, Zoghbi GJ, Perry GJ, Deierhoi M, Warnock D, et al. Predictors of survival in patients with end-stage renal disease evaluated for kidney transplantation. Am J Cardiol 2007;100:1020-5.

20. Kahn MR, Fallahi A, Kim MC, Esquitin R, Robbins MJ. Coronary artery disease in a large renal transplant population: Implications for management. Am J Transplant 2011;11:2665-74.

21. Jones DG, Taylor AM, Enkiri SA, Lobo P, Brayman KL, Keeley EC, et al. Extent and severity of coronary disease and mortality in patients with end-stage renal failure evaluated for renal transplantation. Am J Transplant 2009;9:1846-52.

22. Veroux M, Grosso G, Corona D, Mistretta A, Giaquinta A, Giuffrida G, et al. Age is an important predictor of kidney transplantation outcome. Nephrol Dial Transplant 2012;27:1663-71.

23. Jardine AG, Fellström B, Logan JO, Cole E, Nyberg G, Grönhagen-Riska C, et al. Cardiovascular risk and renal transplantation: Post hoc analyses of the assessment of lescol in renal transplantation (ALERT) study. Am J Kidney Dis 2005;46:529-36.

24. Holdaas H, Fellström B, Cole E, Nyberg G, Olsson AG, Pedersen TR, et al. Long-term cardiac outcomes in renal transplant recipients receiving fluvastatin: The ALERT extension study. Am J Transplant 2005;5:2929-36 